\section{Labordaten- Fernübertragung}

Die Entwicklung der Computertechnologie führt dazu, daß bis Ende der achtziger Jahre alle Arbeiten in Krankenhauslaboratorien, Praxislaboratorien, Forschungslaboratorien usw. computerisiert sein werden. Wenn die Möglichkeiten der Computerisierung nicht nur laborintern sondern auch kommunikationstechnisch genutzt werden sollen, müssen Normen dazu geschaffen werden. Es ist höchste Zeit, mit der Normungsarbeit zu beginnen.

Im Interesse des Laborarztes sollen die Daten so einfach wie möglich in den Computer eingegeben werden, um übersichtliche Computerausgaben am Bildschirm oder ausgedruckt zu erhalten. Für die Verschlüsselung der Informationen im Computer sollte es leicht sein, Normen zu schaffen. Vorteile, die sich daraus ergeben, können an zwei Beispielen erläutert werden:

\section{Beispiel 1}

In einem kleinen Krankenhaus besteht bei einem Patienten der Verdacht auf AIDS. Anamnestische und klinische Daten sowie Laborangaben werden wie üblich erfaßt (in Klarschrift, nach Diktat bzw. im Labor von den Analysengeräten on-line), mittels Akustikkoppler in wenigen Sekunden nicht nur an eine große Universitätsklinik in Deutschland sondern z. B. auch an das Center for Disease Control (CDC) nach Atlanta (USA) zum internationalen AIDS-Zentrum übermittelt, wo der Computerausdruck automatisch in englischer Sprache erscheint. Die aus Atlanta in englischer Sprache gegebene Hinweise für weitere Untersuchungen, Bestätigung der Diagnose, therapeutische Vorschläge erscheinen bei uns dann wieder als deutscher Computerausdruck.

\section{Beispiel 2}

Für eine wissenschaftliche Fragestellung sammelt ein Labor weltweit Analysenergebnisse über die Albumin-, Cholesterin-, Triglycerid--und Glukosekonzentration im Blutplasma, zusammen mit allen notwendigen methodologischen und klinischen Angaben (Geschlecht, Alter, Geburtsort, Wohnort, Beruf usw.), die Ergebnisse aus aller Welt werden über Akustikkoppler oder per Post auf Disketten zugesandt. Wenn alle nötigen methodologischen Angaben mitgeliefert werden, können einwandfreie statistische Analysen erstellt werden. Es kann auch jeder Einzelwert - bei voller Wahrung des Ärztegeheimnisses - zum behandelnden Arzt zurückverfolgt und mit notwendigen weiteren Angaben ergänzt werden.

Weitere Beispiele ließen sich in beliebiger Zahl anführen, um den Sinn und Wichtigkeit einer solchen Normung klarzumachen.

Aufgrund dieser Erkenntnisse hat der DV-Ausschuß von INSTAND nach einer im kleinen Kreis geleisteten Vorarbeit unter Mitwirkung der Commission on World Standards of the World Association of Societies of Pathology (COWS of WASP) zu einem dreiteiligen Symposium im
So können Sie die PMN Elastase bestimmen.

Bei entzündlichen Prozessen ist Elastase aus polymorphnucleären Leukozyten (PMN Elastase) eine krankheitsverstärkende Noxe.

Ihre Bestimmung erlaubt eine frühzeitige Erkennung und eine direkte Verlaufskontrolle einer Entzündung.

Sie wird bestimmt mit dem Merck Immunoassay PMN Elastase, der den Komplex aus PMN Elastase und $\alpha_{1}$-Proteinaseinhibitor erfaßt.

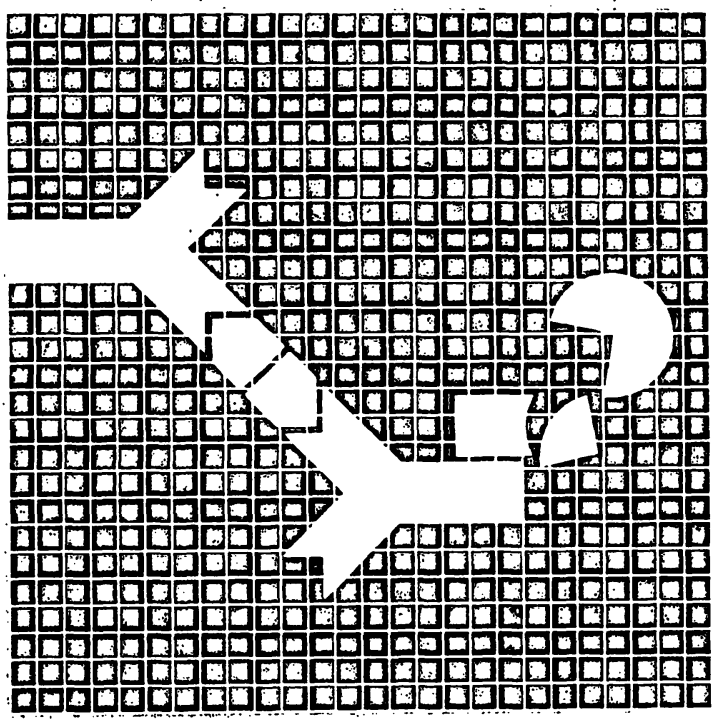

Es handelt sich um einen Festphasenimmunoassay nach dem SandwichPrinzip, der in jedem Labor durchgeführt werden kann.

PMN Elastase - die neue Dimension in der Entzündungsdiagnostik.

Weitere Informationen senden wir lhnen auf Wunsch gerne zu.
E. Merck

Frankfurter Straße 250

D-6100 Darmstadt 1 


\section{UND WAS HALTEN IHRE EINSENDER VON RHRTER METHODE?}

Was Sie und Ihre Einsender von einem CEA-RIA erwarten, ist auf einen gemeinsamen Nenner gebracht, ein Ergebnis, mit dem beide zufrieden sind. Und hier fangen die Unterschiede schon an.

Die meisten CEA-Kit's haben eine mehr oder weniger willkürlich gewählte Kalibration. Und somit jeder Arzt von Labor zu Labor unterschiedlichste Ergebnisse auf dem Tisch. Eine vergleichende Auswertung ist nicht möglich.

Mit dem neuen CEA-RIA von Pharmacia gehört dies der Laborvergangenheit an. Er besitzt eine Kalibration, mit welcher jeder Arzt in seiner täglichen Routine bestens vertraut ist: mit den Standard's der World-Health-Organisation. Kurz: W.H.O. 2/22 J. Diese international eingeführten und vor allem gesicherten Werte gestalten die Diagnose sicherer.

Genauso einfach und präzise gestaltet der Pharmacia CEA-RIA natürlich auch Ihre Arbeit. So z. B. mit seiner immunologischen Trennung, die Ihnen äußerst präzise Ergebnisse liefert. Aber davon mehr in der nächsten Folge oder gleich direkt von Pharmacia unter 0761/49 03-192.

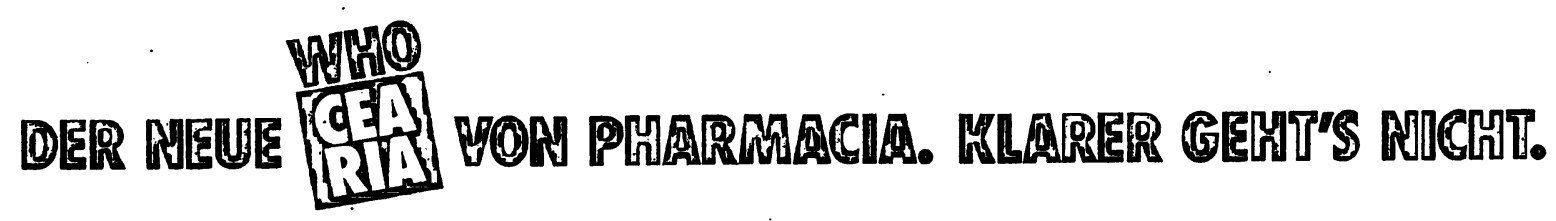


Herbst 1983 in Berlin, Tokio und St. Louis/Ml eingeladen. Die Standorte wurden so ausgewählt, weil die moderne Computertechnologie in Westeuropa, Japan und den USA gleichermaßen Schwerpunkte hat und um den Vertretern dieser drei Regionen die Möglichkeit zur persönlichen Teilnahme zu geben. Aus diesem Grund wurde das Symposium in Tokio im Rahmen des Internationalen Laborärztekongresses und St. Louis/MI während der Herbsttagung des College of American Pathologists gelegt. An den Symposia haben insgesamt 38 Wissenschaftler und Vertreter der Computerindustrie aus Europa. Nord- und Südamerika, Japan und Australien, teilgenommen. INSTAND war durch Dr. daFonseca-Wollheim, Dr. Heller, Dr. Hornscheid, Dipl.-Ing. Kornemann, Dipl.Ing. Kropp, Dr. Lommel, Prof. Dr. R. Merten, Dr. U. P. Merten und Dr. v. Boroviczény vertreten. Den Vorsitz führte in Japan Dr. T. Hutchens, Vorsitzender des COWS of WASP, in Berlin und St. Louis Dr. Elevitch, Chefarzt eines hervorragend computerisierten Krankenhaus-Zentrallabors in Mountain View, Californien, der einen Bericht über dieses Symposium im "Archives of Pathology and Laboratory Medicine" publiziert.

Die vom INSTAND-DV-Ausschuß ausgearbeitete, von R. R. Elevitch und C. R. Elevitch überarbeitete und vor den Sitzungen versandte Vorlage, wurde in Berlin, Tokio und St. Louis jedesmal durchdiskutiert und schrittweise weiterentwickelt, so daß am Ende der ISO (Internationale Normungsorganisation) ein ausgereifter Vorschlag eingereicht werden konnte.

Grundlage des Normenvorschlages ist ein ausgereiftes, in den USA bestehendes und angewandtes Verschlüsselungssystem für alle medizinischen Belange: von der Anamnese über Befund bis zur Katamnese, das SNOMED-Konzept sowie bereits vorhandene internationale Normen zur Datenfernübertragung.

Das Konzept sieht eine Regelung vor, nach der Dateien zur Fernübertragung strukturiert werden sollten: Jedes übermittelte „Paket” besteht aus einem allgemeinen analytorientierten und einem speziellen ergebnisorientierten
Teil. Dabei müssen beide Teile bestimmte Informationen enthalten und können evtl. durch weitere Informationen ergänzt werden. Der allgemeine Teil muß eine Bezeichnung (z.B. Seriennummer), Absenderidentifizierung, Inhaltsverzeichnis und methodologische Angaben enthalten und kann aber auch zusätzliche Anmerkungen übermitteln. Im speziellen Teil müssen Identifizierungscodes und Ergebnisse angegeben werden und nach Wunsch auch weitere Angaben.

Es ist anzunehmen, daß für die Labordaten-Fernübertragung ein "ISO-Standard", d.h. eine International verbindliche Norm, geschaffen wird. Dies wäre ein erster Schiritt auf dem Weg zu einer anwenderorientierten Software-Normung. Mit diesen Fragen befaßt sich der Labordatenausschuß von INSTAND seit einiger Zeit, bei der INSTAND-Herbsttagung soll darüber gesprochen werden. Beim nächsten internationalen Laborärztekongreß im Oktober 1985 ist dafür in Brighton/England ein Symposium geplant. Die Standardisierung der Software ist möglich und sollte auf weltweiter Basis erfolgen, um es für die großen Software-Häuser rentabel zu machen. Erstklassige Konfektion ist viel preiswerter und gleichzeitig besser, als mittelmäßige Einzelanfertigung. Es würde zu einer drastischen Senkung der zur Zeit nahezu unerschwinglichen Software-Kosten führen, das wäre die wichtigste Voraussetzung, um den Computer zum Nutzen des Patienten, aber auch zur Freude und zum Nutzen des Laborpersonals einsetzen zu können.

\section{Anschrift der Verfasser:}

Dr. med. Karl-Georg von Boroviczény

Chefarzt am Zentrallabor-Nord

Krankenhaus Spandau

Lynarstr. 12

D-1000 Berlin 20

Franklin R. Elevitch, M.D.

Director of Pathology Department

El Camin Hosp., 2500 Grant Rd

Mountain View/CA, 94042 USA

INSTAND-Symposium

\section{Organisationsprobleme in der Laboratoriumsmedizin}

\section{Teil V: Laborplanung und Arbeitsablaufplanung}

\section{Ort und Zeitpunkt:}

Düsseldorf, Hotel Nikko (Immermannstr. 41; Tel.: 8661)

Freitag, den 23. November 1984, 14 Uhr bis 19 Uhr

Bustransfer von der Medica, 13.15 Uhr ab Haupteingang

Moderator: Dr. K.-G. v. Boroviczény (Berlin)

\section{Programm:}

\section{Einleitung}

\section{Planung neuer}

Laboratorien

Zur Diskussion eingeladen

Prof. Dr. G. Assmann (Münster)

Dipl. Ing. J. Redmann

(Mannheim)

U. Merten (Düsseldorf)

E. Golz (Berlin)
Investitionsplanung und

Geräteausstellung

Zur Diskussion eingeladen

Allgemeine Diskussion

Kaffeepause

Organisation komplexer

Arbeitsplätze

Zur Diskussion eingeladen

Allgemeine Diskussion

Personaleinsatz bei

Spitzenbelastung und Ausnutzung der

Leerzeiten

Zur Diskussion eingeladen

Allgemeịne Diskussion
Prof. Dr. R. Haeckel (Bremen)

I. Selle (Berlin)

Dr. F. Eßer (Berlin)

Dr. W. Albath (Wiesbaden)

$M$. Söker (Dortmund)

Dr. B. Ostapowicz (Berlin)

Dr. K. Osburg (Hamburg)

Dr, F. G. Weyer (Hannover)

Dr. W. Herold (Berlin)

Kaltes und warmes Buffet (Sponsor dieses Teiles: Boehringer/Mannheim) 